\title{
Design and Devolopment of Smart Framework System for Toddler Monitoring
}

\author{
M. R. Thiyagupriyadharsan, A. Raja Rajeswari, C. Sindhukavi, K. Yogeswaran
}

\begin{abstract}
This paper proposes an efficient monitoring system for toddlers, with wireless communication based on IoT technology. A prototype is developed which gives a reliable and efficient toddler monitoring system that can play an important role in providing better toddler care. This structural set-up monitors and display the essential parameters such as range of temperature and humidity content and heart pulse range in bpm also the movement of a toddler and this information is conveyed to their parents. The main reason for choosing this kind of system for their toddler is to give a comfort and contentment to the loved ones when they are away from their toddler as they can get update status of their well beings. The whole prototype will be working on the basis of a single controller which controls the entire system to provide the precise output extended its advancements to the cloud storage which provides easy access and increased memory and efficiency. The other advantageousness is the scheduling of alarm conditions can take the edge off any imprecision through a conventional sensor. Communication is done by cyberspace which is need and uncomplicated accessible thing.
\end{abstract}

Key words: DHT Sensor, LCD, Sound Sensor, Heartbeat Sensor, Atmega 328P, Wi-Fi module, Wireless Camera

\section{INTRODUCTION}

The toddler observance system could be a variety of warning device which may find babies' movements and activities and may convey the message regarding the condition of babies to the involved authority via a mobile or perhaps a show through a web application .Since from the very first starting of humanity, families have had instincts to secure their babies from probable dangers and risk. The method by which co-parents take care of their youngsters has modified the technological breakthroughs. They are currently pondering adopting the technological and engineering inventions for obtaining benefits and advantages in terms of questions of safety of their babies. During this era once folks are busy with their career, a contemporary baby observance system are often an answer for handling babies properly rather than keeping them in babies' day care center or appointing a baby-sitter for them. Observing a baby unendingly is absolutely a troublesome job still because it isn't potential for the co-parents to hold out their babies all the time with them.

Revised Manuscript Received on March 17, 2020.

* Correspondence Author

Mr. M. R. Thiyagupriyadharsan*, Assistant Professor, Sri Krishna College of Technology

Ms. A. Raja Rajeswari, Student, Bachelor of Engineering, Sri Krishna College of Technology

Ms. C. Sindhukavi, Student, Bachelor of Engineering, Sri Krishna College of Technology

Mr. K. Yogeswaran, Student, Bachelor of Engineering, Sri Krishna College of Technology

(C) The Authors. Published by Blue Eyes Intelligence Engineering and Sciences Publication (BEIESP). This is an open access article under the CC BY-NC-ND license (http://creativecommons.org/licenses/by-nc-nd/4.0/)
Hiring a caretaker for non-stop observance of babies is associate degree and it won't provide a complete solution for this issue. Apart these some ways might not be commodious for co-parents consistent with their demands. Most significantly folks don't get surety regarding their babies' safety in each separate circumstances. During this perspective, a toddler monitor are often the simplest resolution to get rid of the anxiety and stress of the oldsters. During this theisis ,we have got designed a toddler observance system which is rid from victimization. The high performance arithmetic logic unit operates in direct connection with thirty two general purpose registers are executed. Arduino module employed here is UNO R3 and it has the great advantageousness over microcontroller based ones, The baby observance system consists of condenser MIC and it helps in detecting whether the baby is crying or not with different analog range of values and find other essential parameters to take care of the baby safely and it is showed in the external system.

\section{TRADITIONAL PROBLEMS FACED BY TODDLERS:}

During recent era, there occurs a mystery in death of the babies which are especially two to four months old. This as a result, occurs an endangerment of upto the death of 5000 babies specifically in the city-sides of the United States. This phenomenon is termed as Sudden Infant Death Syndrome (SIDS) which is described as the unexpected suffocation of toddler less than one year of age. On the recent survey it is explained as the death which is caused unexpectedly and if that case remains unexplained including the complete performance of autopsy and careful examination of death scene also the review of complete clinical history, it is found that the death attributed to SUID or SIDS which remains the mysterious event for parent or caretaker. Medical skilled people identified that SIDS was complete tragedic and serious scene investigation to exclude the associated factors such as an autopsy which causes upto $90 \%$ of death occurs as an effect for child who are less than six months of age.

Rebreathing Stresses When a child is facing down, air movement around the mouth may be impaired which causes the capture the baby's motion which will be connected to a show such that the baby exhaled Co2.Soft bedding such as blankets, sofas ,soft mattresses and chairs also the other type of sleep surfaces that impair the normal movement of air around the mouth of the baby when nose is positioned down. Hyperthermia This phenomenon of using excessive coverings or increasing the air temperature may lead to an raise in metabolic rate including the overdressing of the toddler causes eventual loss of breathing control.

\section{Published By:}

Blue Eyes Intelligence Engineering DOI: 10.35940/ijeat.C6398.049420

Journal Website: www.ijeat.org

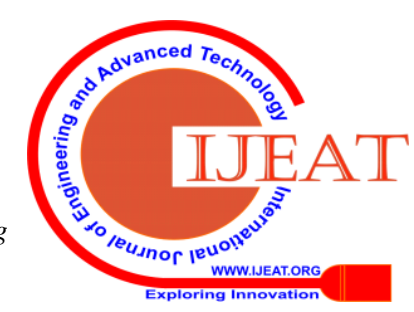

\& Sciences Publication

(C) Copyright: All rights reserved. 
However it is just the external factors which is affecting the infant, those are the objects obstructing the airway due to increased temperature. This is termed as Hyperthermia. This is explained as the major cause for Sudden Infant Death Syndrome(SIDS).
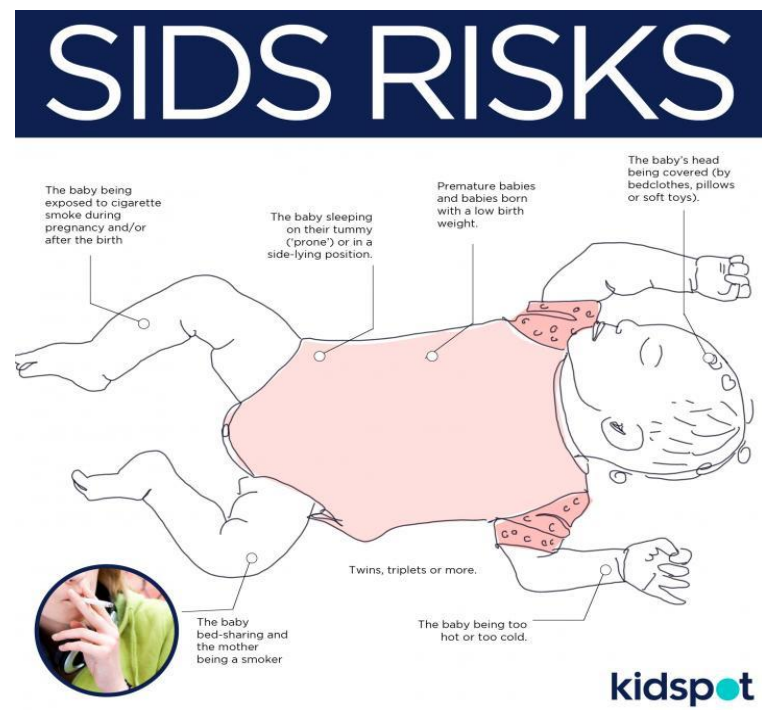

FIG.1. Risks of Sudden Infant Death Syndrome(SIDS).

\section{HARDWARE DESCRIPTION OF THE PROPOSED MODEL:}

The proposed model consists of the following hardware modules:

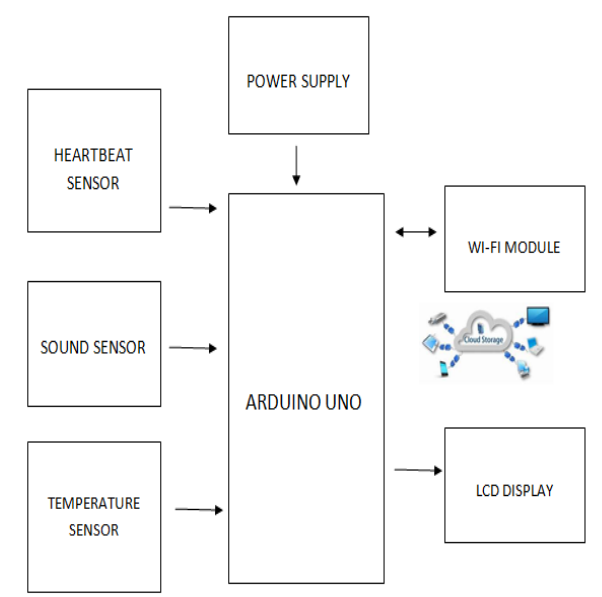

Fig.2. Block diagram of the proposed Toddler Monitoring System.

\section{A. Arduino UNO}

The Arduino UNO has 14 digital pins (six capable of PWM output), 6 analog $\mathrm{I} / \mathrm{O}$ pins, and is programmable with the Arduino IDE (Integrated Development Enviroment), via a type B USB cable. The power is given by external USB cable through $9 \mathrm{~V}$ battery within seven and twenty volts. The ATmega328 on the board comes programmed with a bootloader that allows uploading new code to it without the use of an external hardware programmer.

\section{B. DHT 11 sensor:}

The DHT11 sensor is a basic, digital temperature and humidity sensor. These sensors contain a chip which performs analog to digital conversion and spit out a digital signal with the temperature and humidity. In DHT11 sensor, the humidity sensing component is a moisture holding substrate with the electrodes applied to the surface. This sensor can be easily interfaced with any microcontroller such as arduino to measure humidity and temperature instantaneously. There occurs as a function of both storage of calibration coefficients and transmit the signal which is digital in nature between the arduino and DHT11. It uses one signal wire to transmit data to the arduino

\section{Heartbeat sensor:}

In this system, heartbeat sensor is designed to give digital output of heartbeat when a finger is placed on it for the purpose of monitoring of an infant. The features of heartbeat sensor includes it indicates heartbeat by LED, provides a direct digital signal for connecting to a microcontroller, it possesses compact size and works with a working voltage of $+5 \mathrm{~V}$ DC. The sensor measures the light that get transmitted to the light dependent resistor. In order to calculate the heartrate based on the blood flow to the finger-tip, a heartrate sensor is assembled with the help of LM358 OP-AMP for monitoring the heartbeat pulses.

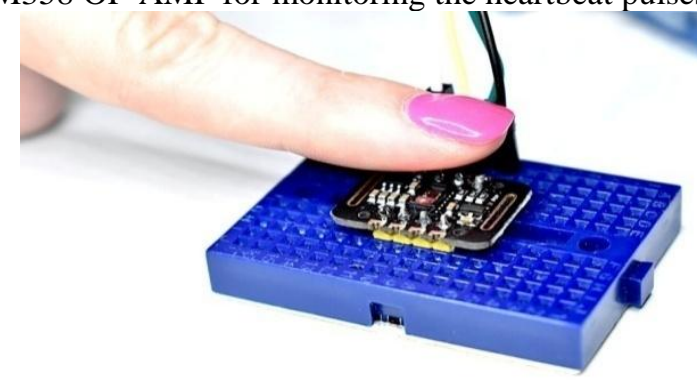

\section{FIG.3. Heartbeat Sensor sensing bpm when the finger placed in the sensor.}

\section{Sound Sensor:}

The sound sensing element is one variety of module won't to notice the sound. Generally, this module is employed to sight the intensity of sound. The applications of this module in the main embody switch, security, in addition as observation. The accuracy of this sending element is modified for the benefit of usage. This sensing element employs a electro-acoustic transducer to produce input to buffer, peak detector associated an electronic equipment. This sensing element notices a sound, \& processes associate output voltage signal to a microcontroller. This sensing element is capable to work out noise levels among DB's or decibels at three rate half dozen rate frequencies some where the human ear is sensitive. In smartphones, there's associate mechanical man application particularly sound unit meter wont to live the sound level.
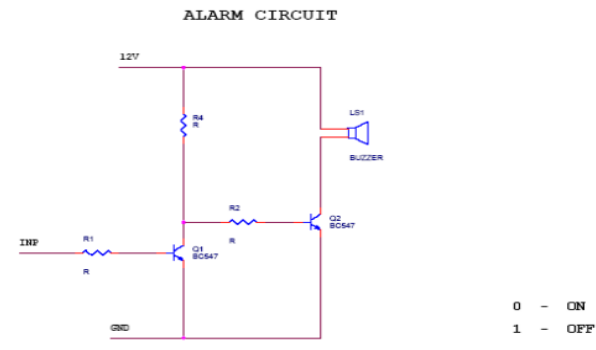

FIG.4. Alarm circuit used in Sound sensor

Published By:

Blue Eyes Intelligence Engineering

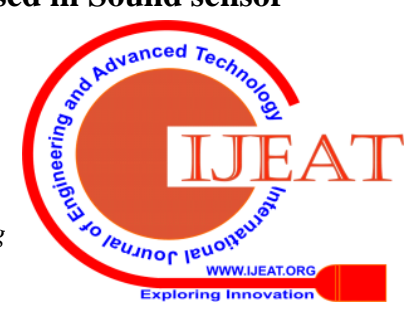




\section{E.IOT Wi-Fi Module:}

The proposed system that consists of $\mathrm{Wi}-\mathrm{Fi}$ module of interrelated computing devices, which are provided with unique identifiers and also the ability to transfer data over a network without requiring human-tohuman and also human-to-computer interaction. The ESP8266 is an Wi-Fi module also available in the system which are self contained SOC with integrated TCP/IP protocol stack which can give any microcontroller access to the Wi-Fi network. The Wi-Fi module is capable of hosting an application and offloading all Wi-Fi networking function from another application processor. This system comprises of a module which allows microcontrollers to connect to a Wi-Fi network. It is connected to arduino by connecting this module to PC, open the arduino, after going to file preferences, adding this link to additional board manager and finally find the module gets activated.

\section{F.LCD DISPLAY:}

LCD display used here in this system is 16*2.Generally, LCD can be used to display information from the arduino and also any sensor connected to it. For example, temperature monitoring system , which displays the temperature on the arduino. When an electricity is passed through them which uses the liquid crystals that become visible and the information is displayed on a LCD screen. An LCD is a highly useful output device for the Arduino. In our proposed system $16 * 2$ LCD module is utilized, having 2 lines and 16 section along these lines add up to 32 characters are shown. This module has two task modes, one uses every one of the 8 pins and alternate uses just 4 of them. The 4 - bit mode was utilized in this system to deal with the LCD screen. All the sensor yield is shown persistently as it is being estimated

\section{METHODOLOGY OF THE PROPOSED MODEL}

Toddler Monitoring System using IoT has three sensors. First one is heartbeat sensor , the second one is temperature sensor, the third one is the sound sensor and the output is displayed in LCD display and all the parameters are consolidated and displayed in an web application. This model is very useful for the parents for observing the child's condition from anywhere in the region outside the home. This allows the user(their parents) to know the health factors of their child such as body temperature, pulse rate, moisture content level in and surrounding the body of the child. This system also detects whether the child is crying or not is intimated by the alarm inside specific region and after the prescribed region which is intimated in the web application at definite intervals of time. These values are stored in the cloud hub storage which is having access to the users via the web application, so that the user can view and recongnise the old and stored date which will be more helpful for the parents and increase their hope in security towards their child. This web application can be accessed by separate URL so that the developed web application can be accessed by the user. This is operating in the principle of connecting the arduino(microcontroller) to the Wi-Fi module by a source of $\mathrm{W}-\mathrm{Fi}$ network . These inputs were read by the sensors and are sent to the cloud by sending the data to the separate IP address and these are processed using arduino. The same sequence of tasks are repeated and displayed over the app. This web application can be easily accessible for laptop and in Smart phones so called the app is portable.

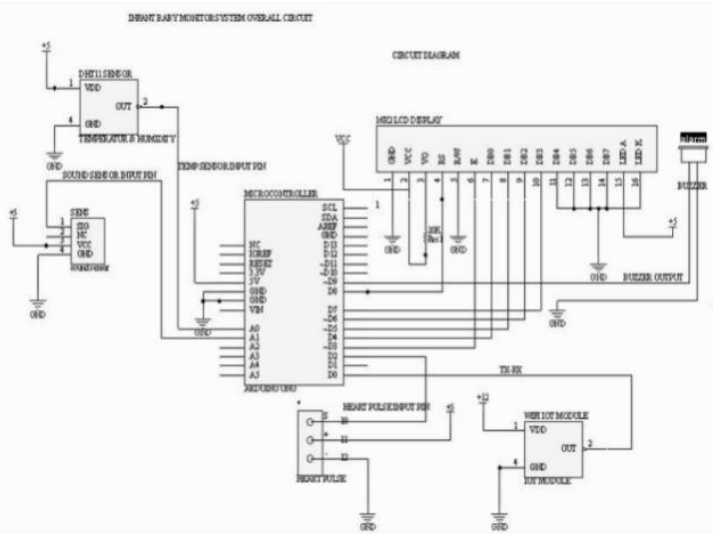

Fig.5.Circuit diagram of Toddler Monitoring System

\section{RESULTS:}

The toddler monitoring system was tested precisely and the system's output was measured. During the execution of the prototype, some snapshots were taken and these were placed in Fig.6,7, 8and 9. The test results of the system were placed in these snapshots which shows the successful implementation of the system prototype. It gives the complete health factors description in a single model via a web application and it is viewed in laptop or smart phones. The readings obtained from this system is compared with standard test instruments such that this prototype is found to be the successful implementation.

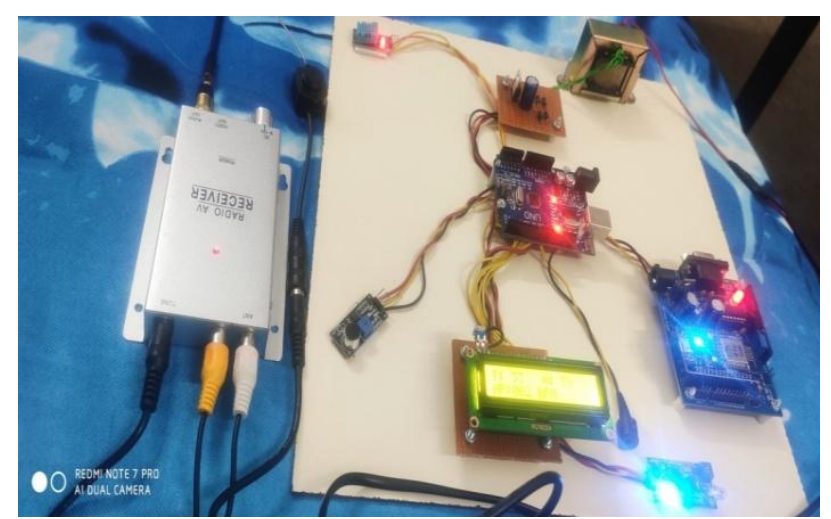

Fig.6.Experimental setup of Toddler Monitoring System

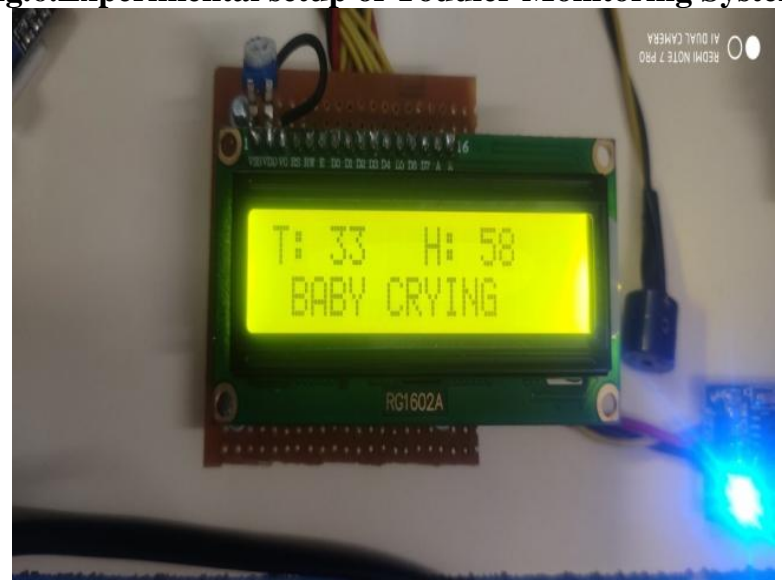

Fig.7.Measuring of temperature and humidity along with displaying whether baby crying or not

Published By:

Blue Eyes Intelligence Engineering

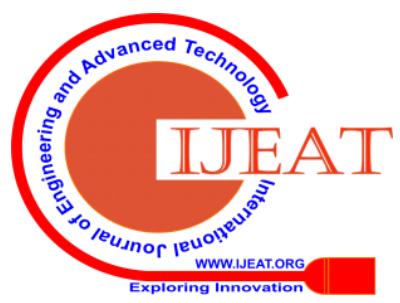




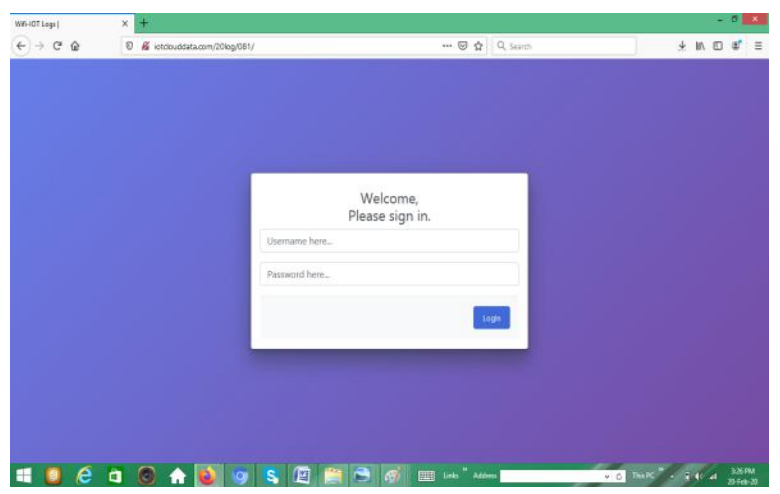

Fig.8.Web application for Toddler Monitoring System.

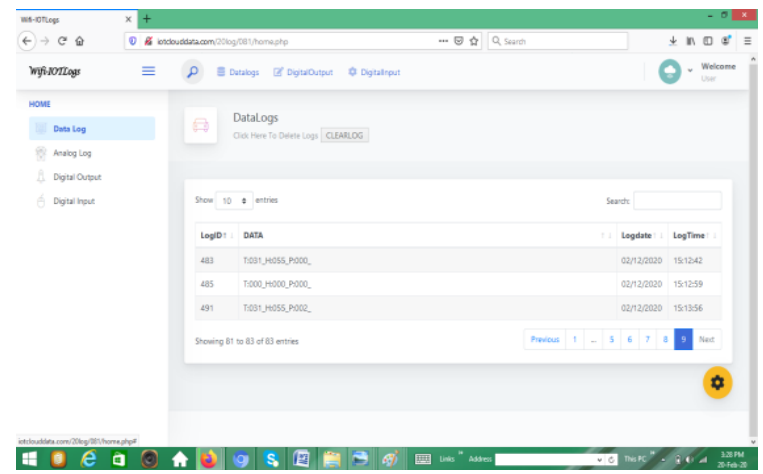

Fig.9.Webpage shows the measured health factors

\section{CONCLUSION}

The toddler monitoring system proposed is to carefully monitorize the movement of the toddler and to keep the toddler in a much safer and security space with an automated observance which helps in reducing energy and manpower in a better way. The results which are taken during the execution of the system is captured and the results are tabulated. Further advancements can be made in the system by providing extra features and setting up the separate web client interface server which will help to provide advanced technology of increased efficiency in the output.The research of robotic cat is undergone in the basis of the toddler monitoring system which will help to serve people of all age-groups especially oldsters.

\section{Table 1. Tabulated Readings Of The Toddler Monitoring}

\begin{tabular}{lllll}
\hline $\begin{array}{c}\text { S.No } \\
\text { Nemperature(value indeg) }\end{array}$ & $\begin{array}{l}\text { Humidityvalue(in } \\
\text { grams of } \\
\text { moisture) }\end{array}$ & $\begin{array}{l}\text { Heartpulserate } \\
\text { (BPM) }\end{array}$ & $\begin{array}{l}\text { Baby Crying } \\
\text { (Yes/No) }\end{array}$ \\
\hline 1 & 34 & 64 & 032 & YES \\
\hline 2 & 33 & 58 & 065 & NO \\
\hline 3 & 31 & 56 & 056 & NO \\
\hline 4 & 32 & 59 & 072 & YES \\
\hline 5 & 35 & 66 & 073 & NO \\
\hline 1 & & & & \\
\hline
\end{tabular}

\section{REFERENCES}

1. Prajakata A.Pawar," Vital sign watching system exploitation IR base device \& Arduino Uno ", IEEE Conference publication on IT business, March 12,2014.

2. Nicec Nithin P. Jain, Preethi N. Jain,and Trupthi P. Agarkaar, "An Embedded, GSM based,Multiparameter,Realtime Patient watching System and Control", IEEE Conference publication in World
Congress on data and Communication Technologies,November 2,2013.

3. Elhaam Saadatian, ShrutiPriyaIyer, subgenus Chen Lihhui, Owen Noel Newton Fernando, Niii Hideaki, Adrian David Cheeok, Ajith Perakum Madurapperuma, Gopalakrishna konee Ponnampalam, and Zubair Amiin,on "Automatic health watching system" command in IEEE International Conference on March four,2016

4. Bakar Muhammad, Hazem Elgabra, Reem Ashur, and Akha Saleih, "Portable Wireless medical specialty Temperature watching System", IEEE international conference publication on innovations in data technology (IIT), nineteen March 2013.

5. Hacking IoT: A Case study on Baby Monitor Exposures and Vulnerabilities by Mark Stainslav and Tod Beardsley printed in International IEEE Conference stayed September 2015.

6. “Advance baby monitor" written by Shrilatha, Shreyaa Paii, Sonaal Cynthiea Pereiraa, Tanya Nicole, Ms.Usha Devi, command in IEEE International Conference of web of Things March 2017

7. "A epitome of kid watching System exploitation motion and authentication with raspberry pi" written by Okky Permatasari,Siti ummy masruoh,Harini command in fourth International IEEE Conference on Cyber and IT Service Management 26-27 Gregorian calendar month 2016

8. "Health watching and Mangement exploitation IoT Sensing and cloud primarily based processing: Oppurtinities and Challenges" by Moen Hasanalieragh, Alex Page,Tolga Syato,Gaurav Sharma stayed IEEE International Conference on Services Computing March 2015

9. M.Lann , k.Sami , N.anusha, M.K.Aiswarya on "A cloud computing resolution on health care institutions" in Second International Conference on E-Health Telemedicine and Social telemedicine"10 Gregorian calendar month 2010

10. T.Soyato, D.Xie C.Funai, R.Muralidharan on "Real time face recongnition exploitation mobile application on advanced devoloped cloud technology" seventeenth IEEE Conference on Gregorian calendar month 2015.

\section{AUTHORS PROFILE}

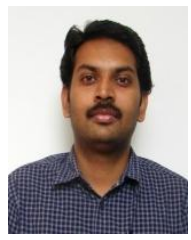

Mr. M. R. Thiyagupriyadharsan, who is currently working as the Assistant Professor in Sri Krishna College of Technology who completed his U.G in V.R.S.College of Engineering and technology and completed his P.G in Coimbatore Institute of Technology and has his area of specialization as Embedded and Real Time Systems

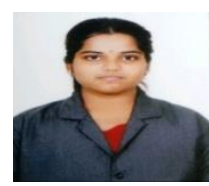

Ms. A. Raja Rajeswari, who is currently pursuing final year in Bachelor of Engineering in Sri Krishna College of Technology with an area of specialization in Embedded Systems.

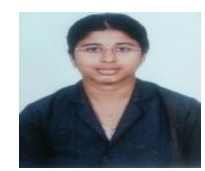

Ms. C. Sindhukavi, who is currently pursuing final year in Bachelor of Engineering in Sri Krishna College of Technology with an area of specialization in Microelectronics design

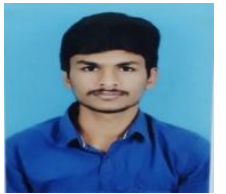

Mr. K. Yogeswaran, who is currently pursuing final year in Bachelor of Engineering in Sri Krishna College of Technology with an area of specialization in Networking.

Blue Eyes Intelligence Engineering

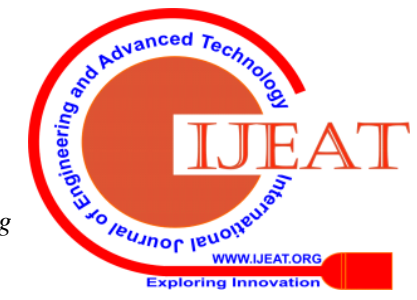

